\title{
Surgical intervention may be a therapeutic option for NSCLC patients with AJCC stage IV: a large population-based study
}

This article was published in the following Dove Press journal:

Cancer Management and Research

\author{
Haiyong Wang ${ }^{1, *}$ \\ Lei Yan ${ }^{1, *}$ \\ Cheng $\mathrm{Li}^{2}$ \\ Zhehai Wang'
}

'Department of Internal MedicineOncology, Shandong Cancer Hospital and Institute, Shandong Cancer Hospital, Shandong University, Shandong Academy of Medical Sciences, Jinan 250 I I7, China; ${ }^{2}$ Department of School of Health Care Management, Shandong University, Key Laboratory of Health Economics and Policy Research, Jinan 250100 , China

*These authors contributed equally to this work
Correspondence: Zhehai Wang Department of Internal MedicineOncology, Shandong Cancer Hospital and Institute, Shandong Cancer Hospital, Shandong University, Shandong Academy of Medical Sciences, Jiyan Road 440, Jinan, Shandong 250117, China

Tel +8653187984777

Fax +8653187984079

Email badgood007@I26.com
Background: Few studies have focused on the role of surgery in the treatment of stage IV nonsmall cell lung cancer (NSCLC). In our study, we mainly focused on the surgical therapeutic option for NSCLC patients with American Joint Committee on Cancer stage IV.

Patients and methods: Using the Surveillance Epidemiology and End Results database, we screened out an appropriate patient population with stage IV NSCLC treated between 2004 and 2013. Kaplan-Meier curve analysis was used to compare the survival between patients receiving surgery and standard of care. The effect of surgery on primary and regional/distant sites on overall survival (OS) was further evaluated by Cox proportional hazard model. Finally, subgroup analysis based on patient and disease variables was conducted by Cox proportional hazard and presented as a forest plot.

Results: A total of 61,418 stage IV NSCLC patients were enrolled. However, only $11.6 \%$ received local surgical treatment. Surgery to primary and regional/distant sites were both independent prognostic factors of OS $(P<0.001)$. Survival advantage was identified in those patients who received surgery to primary sites for all subgroup variables $(P<0.001)$. However, survival benefit was not demonstrated for patients with surgery to regional/distant sites in some subgroup variables, including black racial background, squamous cell carcinoma, large cell carcinoma, and N1 staging (all, $P>0.1$ ). Importantly, we observed that surgery of primary tumor sites at stage N0 showed the maximum OS benefit $(P<0.001)$.

Conclusion: These findings about $\mathrm{N}$ staging and primary tumor site treatment should be taken into consideration by surgeons when determining the suitability of surgery for stage IV NSCLC patients.

Keywords: lung cancer, surgery, stage IV, prognosis

\section{Introduction}

Most patients with non-small cell lung cancer (NSCLC) are found to have distant metastasis at the time of initial diagnosis. ${ }^{1,2}$ The American Society of Clinical Oncology Clinical Practice Guideline Update underscores the fact that there is no cure for stage IV NSCLC, but systemic therapy, such as chemotherapy, targeted therapy, and so on, are recommended as the main treatment modalities. ${ }^{3}$ However, in recent years, due to the improved effect of systemic treatment and further understanding of the concept of "oligometastasis", medical professionals have begun to focus on the role of local therapy in the treatment of NSCLC. ${ }^{4-7}$ Notably, the revised eighth edition of TNM classification dictates that a single metastatic lesion in a single distant organ should be included in the new M1b category; whereas multiple lesions in a single organ or 
multiple lesions in multiple organs should be reclassified as M1c category. ${ }^{8}$ This revision may determine the possibility of local treatment, such as surgical intervention.

In the present study, we mainly focus on the effect of local surgery on primary sites or regional/distant sites on overall survival (OS) for stage IV NSCLC patients. The Surveillance, Epidemiology, and End Results (SEER) database was used to collect relevant patient data for this analysis.

\section{Methods}

\section{Patient selection}

The SEER database, which includes 18 population-based cancer registries, was used to identify appropriate patient records from a large NSCLC population. The SEER program by the National Cancer Institute provides information on cancer statistics in an effort to reduce the cancer burden among the US population. SEER is supported by the Surveillance Research Program, which provides national leadership in the science of cancer surveillance as well as analytical tools and methodological expertise in collecting, analyzing, interpreting, and disseminating reliable population-based statistics. ${ }^{9}$ The SEER * Stat 8.3.4 software was used to screen stage IV NSCLC patients with adenocarcinoma, squamous cell carcinoma, and large cell carcinoma treated between 2004 and 2013. Included patients had been microscopically confirmed NSCLC with only 1 primary tumor. Importantly, all included patients had definitive data about survival and surgery available. In addition, patients before 2004 were excluded due to undetailed staging. Finally, patients with lack of clear information on subgroup variables, including age, race, sex, histology, and radiation were also excluded. A flow diagram of the whole selection process is presented in Figure S1.

Personal identifying information is not included in the SEER database so informed consent was not required. Reference number 11561-Nov2016 was used to access the database and this study was approved by the ethics committee of the Shandong Cancer Hospital affiliated with Shandong University.

\section{Statistical analysis}

OS was regarded as the main study endpoint. Chi-square testing was applied to compare the baseline variables characteristics. The Kaplan-Meier method was used to construct survival curves, and the log-rank test was used to analyze the differences in survival. Univariate and multivariate Cox regression analysis was then used to assess whether surgical intervention was an independent prognostic factor for OS after adjusting for other subgroup variables. Importantly, the effect of local surgery to primary or regional/distant sites on OS using on different subgroup variables, including age, race, sex, histology, stage, and radiation was analyzed by multivariate Cox regression. The resulting statistical information was presented using forest plot. All statistical analyses were performed using SPSS version 22.0 (SPSS, Chicago, IL, USA). All $P$-values were 2 -sided and $P<0.05$ was considered statistically significant.

\section{Results}

\section{Patient demographics}

According to study exclusion and inclusion criteria, a total of 61,418 stage IV NSCLC patients were enrolled. Detailed clinicopathologic features were compared between the patients who underwent surgery and those with no surgical history (Table 1). Among these patients, only $11.6 \%$ received local surgical treatment. Of patients $<65$ years of age, $53.3 \%$ received local surgical treatment and $41.1 \%$ did not undergo surgical treatment. Most patients were of white

Table I Characteristics of NSCLC with AJCC stage IV extracted from SEER Database

\begin{tabular}{|c|c|c|c|}
\hline Variables & Surgery (\%) & No surgery (\%) & $P$-value \\
\hline Age (years) & & & $<0.001$ \\
\hline$<65$ & $3,807(53.3)$ & $22,3 \mid 4(4 \mid . I)$ & \\
\hline$\geq 65$ & $3,330(46.7)$ & $31,967(58.9)$ & \\
\hline Race & & & $<0.001$ \\
\hline White & $5,756(80.6)$ & $42,387(78.1)$ & \\
\hline Black & $875(12.3)$ & $7,064(13.0)$ & \\
\hline Others & $506(7.1)$ & $4,830(8.9)$ & \\
\hline Sex & & & $<0.001$ \\
\hline Female & $3,306(46.3)$ & $24,062(44.3)$ & \\
\hline Male & $3,831(53.7)$ & $30,219(55.7)$ & \\
\hline Histology & & & $<0.001$ \\
\hline Adenocarcinoma & $5,112(71.6)$ & $36,894(68.0)$ & \\
\hline Squamous & I,587 (22.2) & $14,953(27.5)$ & \\
\hline Large cell carcinoma & $438(6.1)$ & $2,434(4.5)$ & \\
\hline T stage & & & $<0.001$ \\
\hline TI & I,385 (19.4) & $5,652(10.4)$ & \\
\hline $\mathrm{T} 2$ & $2,342(32.8)$ & $13,9 \mid 8(25.6)$ & \\
\hline T3 & $455(6.4)$ & $3,385(6.2)$ & \\
\hline T4 & $2,955(4 I .4)$ & $31,326(57.7)$ & \\
\hline N stage & & & $<0.001$ \\
\hline No & $2,620(36.7)$ & $12,212(22.5)$ & \\
\hline $\mathrm{NI}$ & $844(11.8)$ & $4,216(7.8)$ & \\
\hline N2 & $2,771(38.8)$ & $26,598(49.0)$ & \\
\hline N3 & $902(12.6)$ & I I,255 (20.7) & \\
\hline Radiation & & & $<0.001$ \\
\hline Yes & $3,912(54.8)$ & $25,901(47.7)$ & \\
\hline No & $3,225(45.2)$ & $28,380(52.3)$ & \\
\hline
\end{tabular}

Abbreviations: AJCC, American Joint Committee on Cancer; NSCLC, non-small cell lung cancer; SEER, Surveillance Epidemiology and End Results. 
racial background ( $80.6 \%$ and $78.1 \%$, respectively) and were diagnosed with adenocarcinoma for 2 groups $(71.6 \%$ and $68.0 \%$, respectively). Additionally, the percent of patients undergoing surgery was increased by $2 \%$ among female and reduced by $2 \%$ for male patients compared with those with no surgical history. Importantly, the patients with earlier $\mathrm{T}$ and $\mathrm{N}$ stage tumors were more likely to receive surgery $(52.2 \%$ vs $36.0 \%$ for $\mathrm{T} 1-2$ and $48.5 \%$ vs $30.3 \%$ for $\mathrm{N} 0-1$ ). Interestingly, $54.8 \%$ of patients who underwent surgery also received radiation; whereas only $47.7 \%$ of non-surgery patients received radiation. Detailed patient data are presented in Table 1.

\section{Effect of surgery on OS}

Log-rank testing was used to evaluate survival differences. Specifically, Kaplan-Meier curve analysis showed that NSCLC patients who underwent surgery had better OS $(P<0.001)$; the 5-year survival rate was $11.84 \%$ (Figure $1 \mathrm{~A})$. Next, patients receiving surgery were divided into the following groups: surgery to primary and to regional/distant sites. Analysis demonstrated that both surgical subgroups all had significant survival benefit compared with patients who did not receive surgery; the 5-year survival rate was $19.15 \%$ in surgery to primary sites, and $7.58 \%$ in surgery to regional/ distant sites (all, $P<0.001$ ) (Figure 1B, C). Interestingly, survival benefit was more obvious in patients who received surgery to primary sites than those who received surgery to regional/distant sites $(P<0.001)$ (Figure 1D).

In the next stage of analysis, we adjusted for variables, including age, race, sex, histology, stage $\mathrm{T}$, stage $\mathrm{N}$, and radiation. Multivariate Cox regression was applied to analyze prognostic factors on OS. Results showed that surgery to primary and regional/distant sites were all independent prognostic factors affecting OS (hazard ratio [HR]: 2.176; 95\% CI: $2.085-2.271 ; P<0.001$ for surgery to primary sites; HR: $1.129 ; 95 \% \mathrm{CI}: 1.091-1.168 ; P<0.001$ for surgery to regional/distant sites) (Table 2 ).

\section{A}

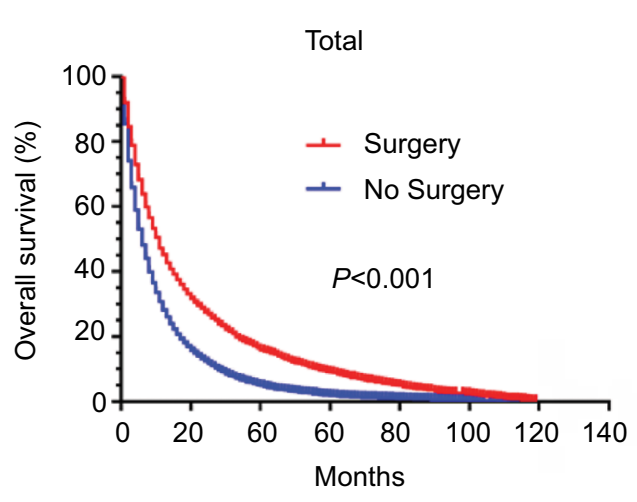

C

Surgery to other regional/distant sites

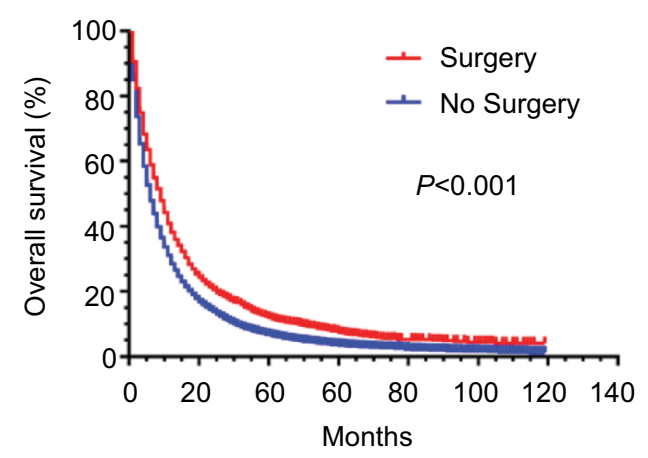

B

Surgery to primary sites

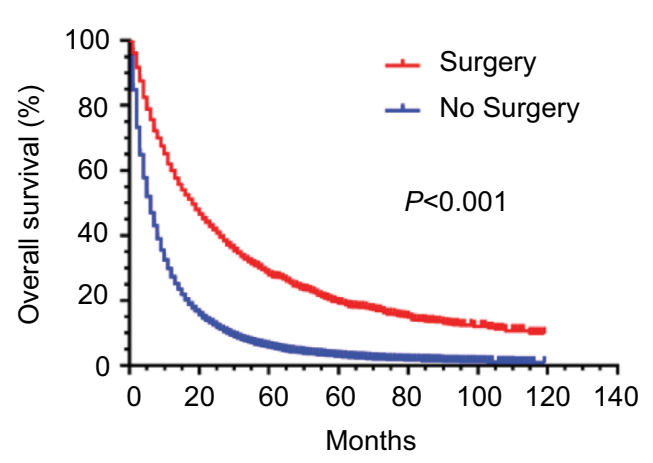

D

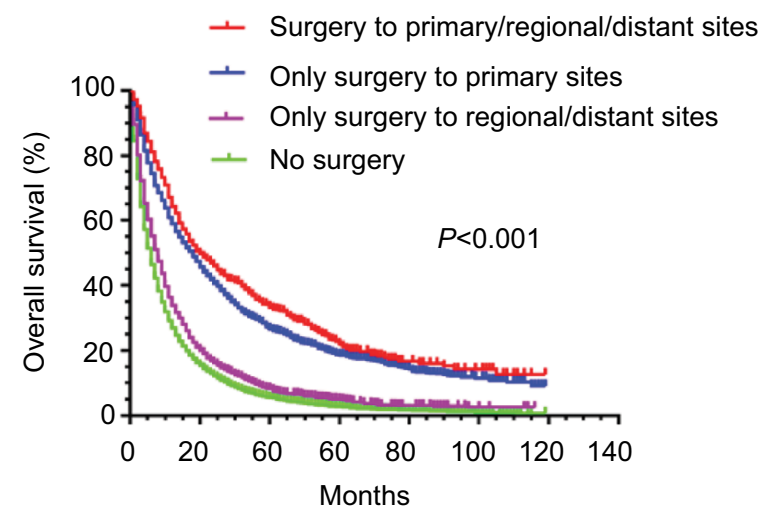

Figure I Kaplan-Meier curves verified by log-rank test.

Notes: (A) Difference in OS between patients who received surgery vs no surgery $(P<0.00 \mathrm{I})$. (B) Difference in OS between patients who received surgery to primary sites vs no surgery $(P<0.00 \mathrm{I})$. (C) Difference in OS between patients who received surgery to regional/distant sites vs no surgery $(P<0.00 \mathrm{I})$. ( $(D)$ Difference in $O S$ between patients who received surgery combination vs no surgery $(P<0.001)$.

Abbreviation: OS, overall survival. 
Table 2 The effect of different subgroup variables on OS for NSCLC with AJCC stage IV analyzed by Cox proportional hazard model

\begin{tabular}{|c|c|c|c|c|}
\hline \multirow[t]{2}{*}{ Variables } & \multicolumn{2}{|l|}{ Univariate analysis } & \multicolumn{2}{|c|}{ Multivariate analysis } \\
\hline & Wald Chi-squared & $P$ & HR (95\% Cl) & $P$-value \\
\hline Surgery to primary sites & $127 \mid .84$ & $<0.001$ & & $<0.001$ \\
\hline Yes & & & Reference & \\
\hline No & & & $2.176(2.085-2.27 I)$ & $<0.001$ \\
\hline Surgery to other sites & 47.54 & $<0.001$ & & $<0.001$ \\
\hline Yes & & & Reference & \\
\hline No & & & 1.129 (1.09I-I.168) & $<0.001$ \\
\hline Age (years) & 562.98 & $<0.001$ & & $<0.001$ \\
\hline$<65$ & & & Reference & \\
\hline$\geq 65$ & & & $1.240(1.2 \mid 8-1.262)$ & $<0.001$ \\
\hline Race & 421.29 & $<0.001$ & & $<0.001$ \\
\hline White & & & Reference & \\
\hline Black & & & $1.015(0.989-1.041)$ & 0.259 \\
\hline Others & & & $0.718(0.696-0.742)$ & $<0.001$ \\
\hline Sex & 437.92 & $<0.001$ & & $<0.001$ \\
\hline Female & & & Reference & \\
\hline Male & & & $1.204(1.183-1.225)$ & $<0.001$ \\
\hline Histology & 267.30 & $<0.001$ & & $<0.001$ \\
\hline Adenocarcinoma & & & Reference & \\
\hline Squamous & & & $1.130(1.108-1.153)$ & $<0.001$ \\
\hline Large cell carcinoma & & & $1.289(1.240-1.34 I)$ & $<0.001$ \\
\hline T stage & 739.26 & $<0.001$ & & $<0.001$ \\
\hline TI & & & Reference & \\
\hline $\mathrm{T} 2$ & & & 1.171 (1.135-1.208) & $<0.001$ \\
\hline T3 & & & 1.338 (1.282-1.397) & $<0.001$ \\
\hline T4 & & & 1.417 (1.377-1.459) & $<0.001$ \\
\hline N stage & 230.40 & $<0.001$ & & $<0.001$ \\
\hline No & & & Reference & \\
\hline $\mathrm{NI}$ & & & $1.092(1.055-1.131)$ & $<0.001$ \\
\hline N2 & & & $1.182(1.156-1.208)$ & $<0.001$ \\
\hline N3 & & & $1.143(1.113-1.174)$ & $<0.001$ \\
\hline Radiation & 92.23 & $<0.001$ & & $<0.001$ \\
\hline Yes & & & Reference & \\
\hline No & & & $1.089(1.070-1.108)$ & $<0.001$ \\
\hline
\end{tabular}

Abbreviations: AJCC, American Joint Committee on Cancer; NSCLC, non-small cell lung cancer; OS, overall survival.

\section{Subgroup analysis of the relationship between surgery and OS}

Multivariate Cox regression analysis demonstrated that variables, including age, race, sex, histology, stage $\mathrm{T}$, stage $\mathrm{N}$, and radiation were all independent prognostic factors of OS. To rule out the effects of these variables and further validate the effect of surgery on OS, we conducted the subgroup analysis based on these variables. Remarkably, obvious survival advantage could be observed in the patients who received surgery to primary sites for all the subgroup variables (all, $P<0.001$ ) (Figure 2). Importantly, patients with surgery to primary sites with stage N0 showed the most significant OS benefit (HR: 0.388 ; 95\% CI: $0.363-0.415, P<0.001$ ); the patients with $\mathrm{N} 3$ also showed the significant OS benefit (HR: 0.736; 95\% CI: 0.631-0.857, $P<0.001$ ) (Figure 2).

Next, we analyzed the effect of surgery to regional/ distant sites on OS taking into account different variables. Survival benefit was not found in patients with surgery to regional/distant sites who were of black racial background, or had either stage N1 squamous cell carcinoma, large cell carcinoma of (black: HR: 0.937; 95\% CI: $0.852-1.031, P=0.181$; squamous cell carcinoma: HR: 0.965 ; 95\% CI: 0.895-1.041, $P=0.361$; large cell carcinoma: HR: 0.977; 95\% CI: 0.857-1.113, $P=0.725$; stage N1: HR: 0.961; 95\% CI: 0.859-1.076, $P=0.490$ ) (Figure 3). Survival benefit was observed among patients 


\begin{tabular}{|c|c|c|c|c|c|c|}
\hline & & & & Surgery to primary sites & & \\
\hline \multirow{3}{*}{ Age } & Variables & Surgery & No & & $\mathrm{HR} \quad(95 \% \mathrm{Cl})$ & $P$-values \\
\hline & $<65$ & 1,578 & 24,542 & ror & $0.450(0.423-0.479)$ & $<0.001$ \\
\hline & $\geq 65$ & 1,714 & 33,584 & юr & $0.448(0.423-0.474)$ & $<0.001$ \\
\hline \multirow[t]{2}{*}{ Race } & White & 2,727 & 45,416 & மต & $0.448(0.428-0.469)$ & $<0.001$ \\
\hline & Black & 357 & 7,581 & $\mapsto$ & $0.458(0.403-0.520)$ & $<0.001$ \\
\hline \multirow[t]{2}{*}{ Sex } & Female & 1,592 & 25,776 & rø- & $0.434(0.407-0.462)$ & $<0.001$ \\
\hline & Male & 1,700 & 32,350 & ror & $0.484(0.457-0.513)$ & $<0.001$ \\
\hline \multirow[t]{3}{*}{ Histology } & $\mathrm{Ad}$ & 2,233 & 39,773 & FH & $0.459(0.436-0.484)$ & $<0.001$ \\
\hline & $\mathrm{Sq}$ & 872 & 15,670 & $1 \bullet-1$ & $0.447(0.412-0.485)$ & $<0.001$ \\
\hline & La & 187 & 2,683 & $\longmapsto$ & $0.530(0.448-0.627)$ & $<0.001$ \\
\hline \multirow[t]{4}{*}{ T stage } & T1 & 564 & 6,474 & $\mapsto$ & $0.395(0.352-0.443)$ & $<0.001$ \\
\hline & T2 & 1,174 & 15,085 & ror & $0.389(0.361-0.419)$ & $<0.001$ \\
\hline & T3 & 287 & 3,554 & $\longmapsto$ & $0.482(0.419-0.556)$ & $<0.001$ \\
\hline & T4 & 1,267 & 33,013 & r๑- & $0.545(0.511-0.581)$ & $<0.001$ \\
\hline \multirow[t]{4}{*}{$\mathrm{N}$ stage } & No & 1,519 & 13,313 & юr- & $0.388(0.363-0.415)$ & $<0.001$ \\
\hline & $\mathrm{N} 1$ & 509 & 4,550 & $\mapsto \bullet$ & $0.437(0.391-0.489)$ & $<0.001$ \\
\hline & N2 & 1,062 & 28,308 & $\mapsto-1$ & $0.534(0.498-0.573)$ & $<0.001$ \\
\hline & N3 & 202 & 11,955 & $\longmapsto$ & $0.736(0.631-0.857)$ & $<0.001$ \\
\hline \multirow[t]{2}{*}{ Radiation } & Yes & 1,321 & 28,492 & r-1 & $0.541(0.507-0.577)$ & $<0.001$ \\
\hline & No & 1,971 & 29,634 & •r| & $0.417(0.394-0.442)$ & $<0.001$ \\
\hline \multirow[t]{2}{*}{ Total } & & 3,292 & 58,126 & ॥ & $0.460(0.440-0.480)$ & $<0.001$ \\
\hline & & & & 0.5 & 1.5 & \\
\hline
\end{tabular}

Figure 2 The effect of primary site surgery on OS based on different subgroup variables.

Notes: Findings were verified by Cox proportional hazard analysis and presented as a forest plot.

Abbreviations: OS, overall survival; Ad, adenocarcinoma; Sq, squamous; La, large cell carcinoma.

with surgery to regional/distant sites in other subgroup variables (all, $P<0.01$ ) (Figure 3).

\section{Discussion}

This study was focused on the effect of surgery on survival in stage IV NSCLC patients. Three interesting clinical findings were demonstrated and will be discussed as follows: local surgery might be an option for stage IV NSCLC patients; surgery to primary sites had better survival benefit compared with patients undergoing surgery at regional/distant tumor sites; and finally, patients with stage N0 may be more suited to receive surgery to primary sites.

Previously, there has not been sufficient evidence on the role of surgery therapy for stage IV NSCLC patients. Some oncologists have focused on this key clinical issue but the issue remains controversial. A single-center study performed in China demonstrated that surgical treatment of M1a NSCLC was associated with improved survival compared with published reports and could be considered in the management of selected cases..$^{10}$ In addition, some studies have shown that selected patients who undergo surgical resection for a primary tumor and receive effective local therapy for metastatic lesions still have a chance to obtain long-term survival. Further studies should focus on the possible use of surgery as a strategy for therapeutic "consolidation" in appropriately selected patients with stage IV NSCLC receiving combined modality care. ${ }^{11,12}$ Another study from Duke University Medical Center demonstrated that surgery for cT1-2, N0-1, M1 or cT3, N0, M1 NSCLC tumors is associated with a 5-year survival of $25 \%$ and does not appear to compromise outcomes when compared with non-operative therapy ${ }^{13}$ Consistent with these results, the present study showed that OS benefit was observed in NSCLC patients who received surgery therapy. Notably, we found that patients who received surgery only to primary sites had better survival prognosis than those who received surgery to regional/distant sites. In fact, few studies have compared survival of patients with only surgery to primary sites vs regional/distant sites.

Several studies have demonstrated that pulmonary resection could bring reasonable long-term survival benefit for patients with oligometastases disease, such as single adrenal or brain metastasis. ${ }^{14-17}$ It should be noted that these studies were based on small sample sizes; however, similar results were validated in this present study based on a large sample 


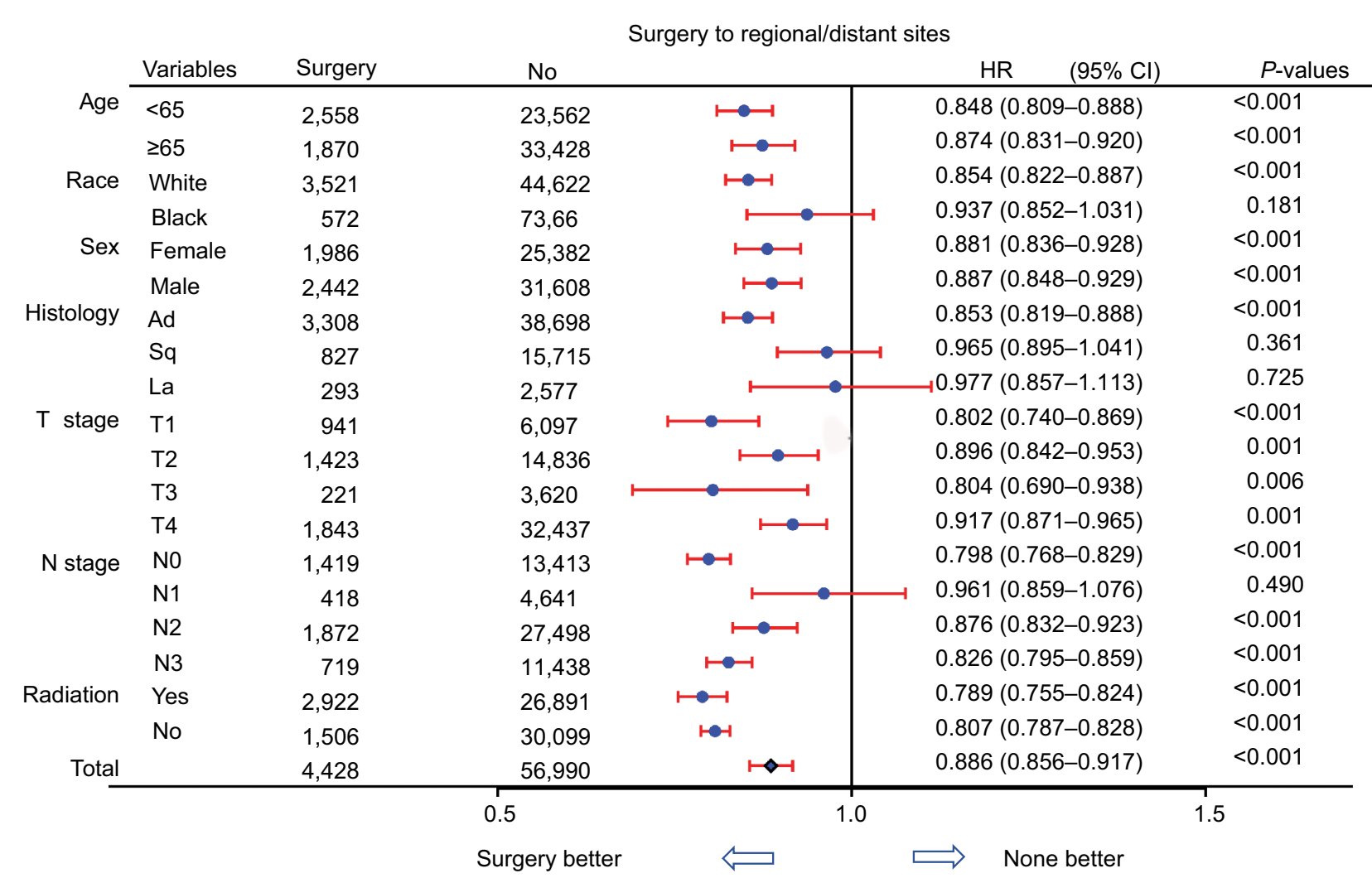

Figure 3 The effect of regional/distant site surgery on OS based on different subgroup variables. Notes: Findings were verified by Cox proportional hazard analysis and presented as a forest plot. Abbreviations: OS, overall survival; Ad, adenocarcinoma; Sq, squamous; La, large cell carcinoma.

size. Some studies also demonstrated that surgical resection of distant sites, including brain, liver, adrenal gland, and other sites could improve the survival rates for such patients..$^{18-20}$ Interestingly, some studies have demonstrated that some select patients may benefit from surgical treatment of both the primary and metastasis sites. ${ }^{21,22}$ In the present study, survival benefit was also found in patients who received surgery to primary and metastasis sites. However, an obvious difference was not found between the patients with surgery to primary/regional/distant sites and those with surgery only to primary tumor sites; therefore, it is still important to study the proper determination of operation sites in for stage IV NSCLC patients.

A review by Novoa et al found that an absence of mediastinal lymph node involvement is a positive predictor of surgery outcomes for stage IV NSCLC patients. ${ }^{23}$ In fact, metastatic N0 NSCLC patients had a favorable prognosis and a lower ratio of adrenal gland metastasis than those with clinical N1-three disease..$^{24}$ In this study, we found that patients with stage $\mathrm{N} 0$ are more likely to benefit from surgery. Several other studies support these findings. Billing et al demonstrated that the presence of thoracic lymph node metastases (N1 or N2) significantly affected 5 -year survival $(P=0.001)$, and surgical resection may prove beneficial in a selected group of patients with synchronous brain metastases and lung cancer without lymph node metastases. ${ }^{25}$ Also, David et al showed that surgical resection of NSCLC can result in a 5-year survival rate of $30 \%-50 \%$, but this figure is heavily influenced by the presence of mediastinal nodal disease, a factor, which must be evaluated before selection for surgery. ${ }^{12}$ Taking into account these results, surgical intervention would bring more benefit to patients with stage N0 NSCLC.

Several limitations should be noted in this study. First, we cannot avoid the impact of selection bias on some baseline variables in this retrospective study, despite the relatively rather large sample size. Second, in multivariate Cox regression analysis, we only analyzed radiation therapy. The other therapy information, including chemotherapy, molecule-targeted therapy, and exact surgical types were not entered in our present study due to the limitations of the SEER database itself. Finally, a study by Hanagiri et al demonstrated that surgical treatment for NSCLC with oligometastatic disease should be considered to be one arm of multidisciplinary treatment. ${ }^{11}$ Some studies also showed that other variables, including good performance status 
and nonsmoking histology may predict for improved survival in such patients. ${ }^{10}$ However, also due to the data limitations of the SEER database, these variables, including good performance status, nonsmoking histology, and the oligometastatic variables were not able to be analyzed in this study.

\section{Conclusion}

Our results showed that selected stage IV NSCLC patients may benefit from surgery of primary tumor sites. Patients especially with stage N0 tumors should be recommended to undergo operation. Multi-institutional trials are warranted to further validate our conclusions and determine the most suitable patients for surgery.

\section{Acknowledgments}

This study was supported jointly by the National Natural Science Foundation of China (No. 81603348); China Postdoctoral fund (No. 21300075311104) and Shandong postdoctoral innovation special fund (No. 201602012); China Postdoctoral Special Fund (No. 2018T110696); Shandong Province key R \& D Plan (2018GSF119014). Haiyong Wang and Lei Yan are co-first authors.

\section{Disclosure}

The authors report no conflicts of interest in this work.

\section{References}

1. Ramalingam S, Belani C. Systemic chemotherapy for advanced nonsmall cell lung cancer: recent advances and future directions. Oncologist. 2008;13(Suppl 1):5-13.

2. Jemal A, Siegel R, Xu J, Ward E. Cancer statistics, 2010. CA Cancer J Clin. 2010;60(5):277-300.

3. Hanna N, Johnson D, Temin S, et al. Systemic Therapy for Stage IV NonSmall-Cell Lung Cancer: American Society of Clinical Oncology Clinical Practice Guideline Update. J Clin Oncol. 2017;35(30):3484-3515.

4. Hellman S, Weichselbaum RR. Importance of local control in an era of systemic therapy. Nat Clin Pract Oncol. 2005;2(2):60-61.

5. Congedo MT, Cesario A, Lococo F, et al. Surgery for oligometastatic non-small cell lung cancer: long-term results from a single center experience. J Thorac Cardiovasc Surg. 2012;144(2):444-452.

6. de Vin T, Engels B, Gevaert T, Storme G, de Ridder M. Stereotactic radiotherapy for oligometastatic cancer: a prognostic model for survival. Ann Oncol. 2014;25(2):467-471.

7. Ashworth AB, Senan S, Palma DA, et al. An individual patient data metaanalysis of outcomes and prognostic factors after treatment of oligometastatic non-small-cell lung cancer. Clin Lung Cancer. 2014;15(5):346-355.
8. Detterbeck FC, Boffa DJ, Kim AW, Tanoue LT. The Eighth Edition Lung Cancer Stage Classification. Chest. 2017;151(1):193-203.

9. Surveillance, Epidemiology, and End Results (SEER) Program. SEER*Stat Database: Incidence - SEER 18 Regs Research Data + Hurricane Katrina Impacted Louisiana Cases, Nov 2015 Sub (19732013 varying) - Linked To County Attributes - Total U.S., 1969-2014 Counties, National Cancer Institute, DCCPS, Surveillance Research Program, Surveillance Systems Branch, released April 2016, based on the November 2015 submission. Available from: https://seer.cancer.gov/.

10. Liu T, Liu H, Wang G, Zhang C, Liu B. Survival of M1a Non-Small Cell Lung Cancer Treated Surgically: A Retrospective Single-Center Study. Thorac Cardiovasc Surg. 2015;63(7):577-582.

11. Hanagiri T, Takenaka M, Oka S, et al. Results of a surgical resection for patients with stage IV non--small-cell lung cancer. Clin Lung Cancer. 2012;13(3):220-224.

12. David EA, Clark JM, Cooke DT, Melnikow J, Kelly K, Canter RJ. The Role of Thoracic Surgery in the Therapeutic Management of Metastatic Non-Small Cell Lung Cancer. JThorac Oncol. 2017;12(11): $1636-1645$.

13. Yang CJ, Gu L, Shah SA, et al. Long-term outcomes of surgical resection for stage IV non-small-cell lung cancer: A national analysis. Lung Cancer. 2018;115:75-83.

14. Yoshinaga Y, Enatsu S, Iwasaki A, Shirakusa T. Surgical treatment for primary non-small cell lung cancer with synchronous brain metastases. Kyobu Geka. 2006;59(1):41-45.

15. Mordant P, Arame A, de Dominicis F, et al. Which metastasis management allows long-term survival of synchronous solitary M1b non-small cell lung cancer? Eur J Cardiothorac Surg. 2012;41(3):617-622.

16. Porte H, Siat J, Guibert B, et al. Resection of adrenal metastases from non-small cell lung cancer: a multicenter study. Ann Thorac Surg. 2001;71(3):981-985.

17. Furák J, Troján I, Szöke T, et al. Lung cancer and its operable brain metastasis: survival rate and staging problems. Ann Thorac Surg. 2005;79(1):241-247.

18. Schuchert MJ, Luketich JD. Solitary sites of metastatic disease in nonsmall cell lung cancer. Curr Treat Options Oncol. 2003;4(1):65-79.

19. Bonnette P, Puyo P, Gabriel C, et al. Surgical management of nonsmall cell lung cancer with synchronous brain metastases. Chest. 2001;119(5):1469-1475.

20. Gaspar LE. Brain metastases in lung cancer. Expert Rev Anticancer Ther. 2004;4(2):259-270.

21. Kawano D, Takeo S, Katsura M, Tsukamoto S, Masuyama E, Nakaji Y. Surgical treatment of stage IV non-small cell lung cancer. Interact Cardiovasc Thorac Surg. 2012;14(2):167-170.

22. Tönnies M, Pfannschmidt J, Bauer TT, Kollmeier J, Tönnies S, Kaiser D. Metastasectomy for synchronous solitary non-small cell lung cancer metastases. Ann Thorac Surg. 2014;98(1):249-256.

23. Novoa NM, Varela G, Jiménez MF. Surgical management of oligometastatic non-small cell lung cancer. J Thorac Dis. 2016;8(Suppl 11):S895-S900.

24. Tamura T, Kurishima K, Watanabe H, et al. Characteristics of clinical N0 metastatic non-small cell lung cancer. Lung Cancer. 2015;89(1): 71-75.

25. Billing PS, Miller DL, Allen MS, Deschamps C, Trastek VF, Pairolero PC. Surgical treatment of primary lung cancer with synchronous brain metastases. J Thorac Cardiovasc Surg. 2001;122(3):548-553. 


\section{Supplementary material}

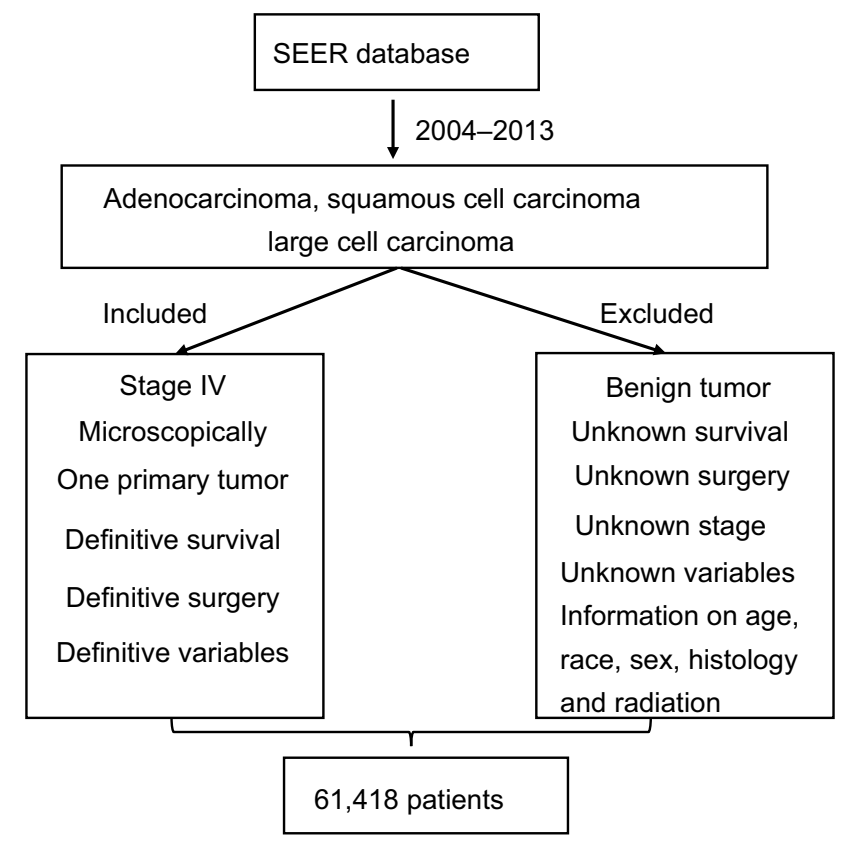

Figure SI Data filter flow chart based on SEER database.

\section{Publish your work in this journal}

Cancer Management and Research is an international, peer-reviewed open access journal focusing on cancer research and the optimal use of preventative and integrated treatment interventions to achieve improved outcomes, enhanced survival and quality of life for the cancer patient. The manuscript management system is completely online and includes a very quick and fair peer-review system, which is all easy to use. Visit http://www.dovepress.com/testimonials.php to read real quotes from published authors. 